\title{
Cost-Effectiveness of the treatment protocols of pediatrics with Acute Lymphoblastic Leukemia (Decision tree modeling)
}

\author{
hadi hayati ${ }^{1}$ and Mohammadali Ehsani ${ }^{2}$ \\ ${ }^{1}$ Lorestan University of Medical Sciences \\ ${ }^{2}$ Tehran University of Medical Sciences
}

February 19, 2022

\begin{abstract}
Acute lymphoblastic leukemia (ALL) has a high prevalence at early age and in children and regard to life expectancy index this disease cause to losing many years of life in these patients. Two well-known European protocols called United Kingdom (UK-ALL) and Berlin-Frankfurt-Munster (BFM-ALL) protocols are used to treat the disease in Iran, so the objective of this study is modelling of the treatment process using the protocols of BFM-ALL and UK-ALL to estimate more cost-effectiveness method. A decision tree model was applied to depict the real treatment process to calculate costs per quality-adjusted life-year (QALY). Total costs were included to the model. The cost effectiveness ratio of UK-ALL is lower than BFM-ALL (1145.52 USD /3.87 QALY for UK-ALL and 1942.35 USD /3.02 QALY for BFM-ALL). Therefore, the UK-ALL is dominant and BFM-ALL is dominated. The modeling results showed a double difference between the two studied options so policymakers and oncologist should advocate the economic evaluation methods and modeling to select a real option in the treatment of patients to save resources like UK-ALL.
\end{abstract}

Cost-Effectiveness of the treatment protocols of pediatrics with Acute Lymphoblastic Leukemia (Decision tree modeling)

\section{A B S T R A C}

Acute lymphoblastic leukemia (ALL) has a high prevalence at early age and in children and regard to life expectancy index this disease cause to losing many years of life in these patients. Two well-known European protocols called United Kingdom (UK-ALL) and Berlin-Frankfurt-Munster (BFM-ALL) protocols are used to treat the disease in Iran, so the objective of this study is modelling of the treatment process using the protocols of BFM-ALL and UK-ALL to estimate more cost-effectiveness method. A decision tree model was applied to depict the real treatment process to calculate costs per quality-adjusted life-year (QALY). Total costs were included to the model. The cost effectiveness ratio of UK-ALL is lower than BFM-ALL (1145.52 USD /3.87 QALY for UK-ALL and 1942.35 USD /3.02 QALY for BFM-ALL). Therefore, the UK-ALL is dominant and BFM-ALL is dominated. The modeling results showed a double difference between the two studied options so policymakers and oncologist should advocate the economic evaluation methods and modeling to select a real option in the treatment of patients to save resources like UK-ALL.

Keywords: Cost, Acute Lymphoblastic Leukemia, Children, Cost- Effectiveness analysis, Decision Tree modelling, BFM-ALL, UK-ALL, QALY.

\section{Introduction}

Acute lymphoblastic leukemia (ALL) have a high prevalence at early age and in children and regard to life expectancy index this disease cause to losing many years of life in these patients [1, 2]. Hence in many countries due to its high cost and complex and prolonged treatment process, important work has 
been done to treat it and it was converted from an incurable to chronic disease like other diseases [3, 4]. The childhood treatment of ALL usually involves chemotherapy for 2 to 3 years, and generally therapies have at least three phases $[1,3,5]$, in this regard the cost issue is an essential factor in the application of these therapeutic protocols and significantly long economic reports and studies have been carried out on easts of these disease, which suggests a huge difference in term of cost between treatments protocols of all indifferent countries and expenses rang in different countries are different with specific protocols and varies from $\$ 6000$ in China to over $\$ 16000$ in America and Europe [6-8]. Also, the success of therapy in various studies has been evaluated in terms of various clinical indicators. Therefore, in Iran two European well known protocols called United Kingdom (UK-ALL) and Berlin-Frankfurt-Munster (BFM-ALL) protocols are used to treat the disease [4], these protocols consists of three phases to treatment include 1)Induction of remission, 2)Consolidation/Intensification and Reinduction and 3) Maintenance[4]. There has never been a research of this kind for proper policy making related to modeling the treatment process and effectiveness and cost of these two protocols Therefore, it seemed necessary to modeling treatment process of this disease in regard to costs and effectiveness in two protocols [9-11]. On the other hand, decision tree analysis is the application of an analytical method of modeling for systematically comparing different decision options. Decision tree graphically shows selections and facilitates the calculation of values needed to compare these choices. It supports with selecting the most cost-effective selection [10, 12]. Decision tree is a tool that has been used for years in many fields. This method of analysis assists in making decisions when the decision is not simple and there is uncertainty about some of the information. So we used this method to compare these two protocols [9].

\section{Methods}

This research is a retrospective study for cost effectiveness modeling. Data were extracted based on hospital information for two protocols UK-ALL and BFM-ALL which are performed for children treatment with ALL in important medical centers in Tehran, the capital of Iran. By initial reviewing the number of the people in the research environment and evaluating the criteria for entry and exit of individuals to study to achieve acceptable results all patients which have proper conditions to enter study were selected as samples. The perspective of these of this study has been society therefore all costs are recognized, identified, measured and valued from this perspective. Three data from community perspective are required to perform modeling methods:

\section{Transition probability}

First, data that involves clinical events, these probabilities used in different branches of decision tree, all death and relapse rates and recovery rates were determined and calculated in each phase by collecting accurate data and in accordance with the treatment process and the options ahead (decision tree), calculated probabilities are mentioned.

\section{Quality adjusted life years}

Second, data from effectiveness of treatment in the form of QALY (quality adjusted life years) were calculated. so that QALY in the all phases of treatment protocols is estimated based on quality of life models which measured by related the EQ5D questionnaire. Finally, according to the scores of Iran, the utility states and QALY were calculated at the end of each phase of treatment.

\section{COSTS}

Third, in relation to cost data include total direct cost of patients (clinical and non-clinical) and indirect cost which include parent child costs, are estimated based on all inputs used to treat patients and their prices. There was comprehensive medical information available at the selected hospitals and by referring to patient's files, from the accounting records in the hospitals were collected.

Hence, a therapeutic process that takes about 4-5 years to complete recovery, were called in five years. All the above information was adjusted to the year 2022 according to the discount rate and the official inflation rate of Iran. To analyze the information at final stage the Treeage 11 software was used. 


\section{Model assumptions}

- According to clinical evidences and specialist opinion and resource review, patients who recovered, five years after start of treatment is considered a perfectly improved person (Remission).

- Considering the decrease in relapsed patients during treatment and insignificant number of them at the end of the treatment or their complete recovery were eliminated from model to improve the results.

- The quality of all hospital care and care at home is considered to be the same for all patients. And excess household expenses and care were excluded from study.

\section{Treatment process}

Figure 1 shows that at first, patients are in the initial phase (Induction). And after the end of this phase they will enter the next phase (Consolidation) and if the treatment process performs successfully, they will come to the next stage (Maintenance) and recovery (Remission). But during treatment there is a possibility of relapse and death in each phase.

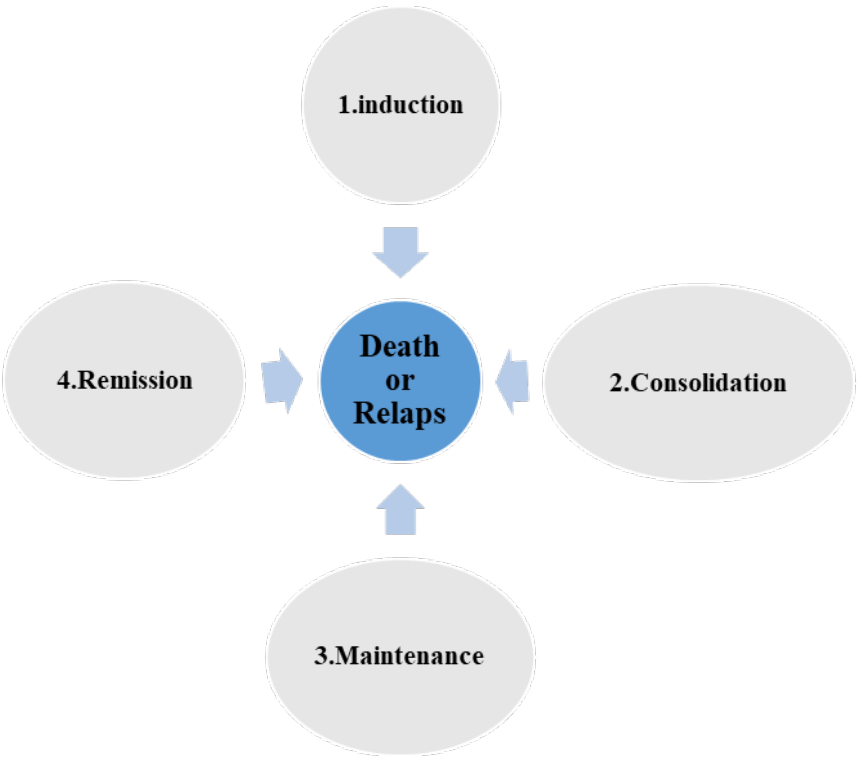

Figure 1: Schematic view of three phases of treatment process

\section{Results :}

According to the results as table 1 shows that after the end of each phase, patients are confronted with a possible death or recurrence or success in entering to the next phase. In each stage the probability of success is very high and only a small number of patient encounter failure but in the final phase morality and recurrence rates are higher than the previous phases.

Table 1 The transition probabilities between the phases of UK -ALL and BFM-ALL

\begin{tabular}{llllll}
\hline & $\begin{array}{l}\text { Success } \\
\text { probability to } \\
\text { next phase (\%) }\end{array}$ & $\begin{array}{l}\text { Success } \\
\text { probability to } \\
\text { next phase (\%) }\end{array}$ & $\begin{array}{l}\text { Success } \\
\text { probability to } \\
\text { next phase (\%) }\end{array}$ & $\begin{array}{l}\text { Death\& } \\
\text { relapse\%) }\end{array}$ & sum \\
\hline $\begin{array}{l}\text { Phase (UK-ALL) } \\
\text {-start of } \\
\text { treatment }\end{array}$ & Consolidation & UK & 97.9 & 2.1 & 1 \\
& & BFM & 92.9 & 7.1 & 1
\end{tabular}




\begin{tabular}{llllll}
\hline & $\begin{array}{l}\text { Success } \\
\text { probability to } \\
\text { next phase (\%) }\end{array}$ & $\begin{array}{l}\text { Success } \\
\text { probability to } \\
\text { next phase (\%) }\end{array}$ & $\begin{array}{l}\text { Success } \\
\text { probability to } \\
\text { next phase (\%) }\end{array}$ & $\begin{array}{l}\text { Death\& } \\
\text { relapse\%) }\end{array}$ & sum \\
\hline $\begin{array}{l}\text { Phase (UK-ALL) } \\
\text { end of } 1 \\
\text { month }\end{array}$ & Maintenance & UK & 97.3 & 2.7 & 1 \\
$\begin{array}{l}\text { Maintenance- } \\
\text { end of } 2.5\end{array}$ & Remission & BFM & 95.4 & 4.6 & 1 \\
years & UK & 94 & 6 & 1 \\
& & & & \\
\hline
\end{tabular}

Also, table 2 shows that the cost and QALY of each phase of the protocols separately, and as seen, for UKALL the total cost is lower than BFMALL and also the QALY is more.

Table 2 the cost and QALY of each phase of UK -ALL and BFM-ALL protocols

\begin{tabular}{lllll}
\hline Phase & Induction & Consolidation & Maintenance & Total \\
\hline QALY- BFM & 0.05 & 0.12 & 3.23 & 3.40 \\
Cost (USD) & 733.00 & 776.24 & 549.75 & 2059 \\
Percent & 0.356 & 0.377 & 0.267 & 1 \\
QALY- UK & 0.04 & 0.02 & 4 & 4.06 \\
Cost (USD) & 460.60 & 275.42 & 435.98 & 1172 \\
Percent & 0.393 & 0.235 & 0.372 & 1 \\
\hline
\end{tabular}

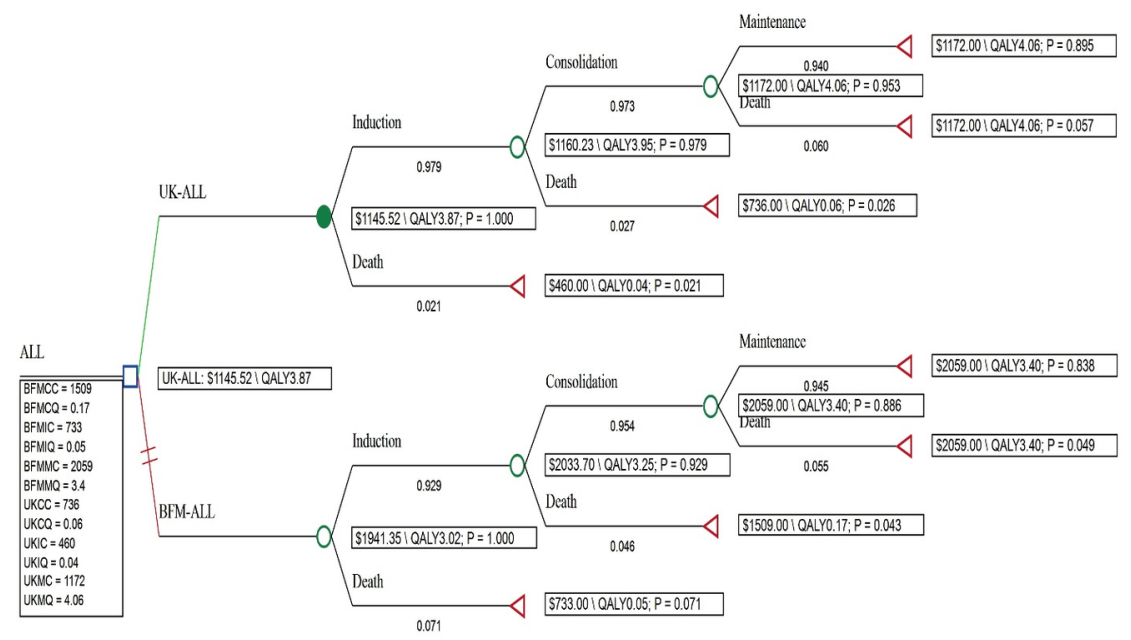

Figure 2 Decision tree modeling of pediatric ALL treatment protocols

Figure 2 shows the results of the decision tree analysis, finally the cost effectiveness ratio of UKALL is lower than BFMALL so that means less money per QALY pay for UKALL than BFMALL. $(1145.52 / 3.87=296$ UKALL and 1942.35/3.02=643.2). It indicates a double difference in this ratio in these two methods. 


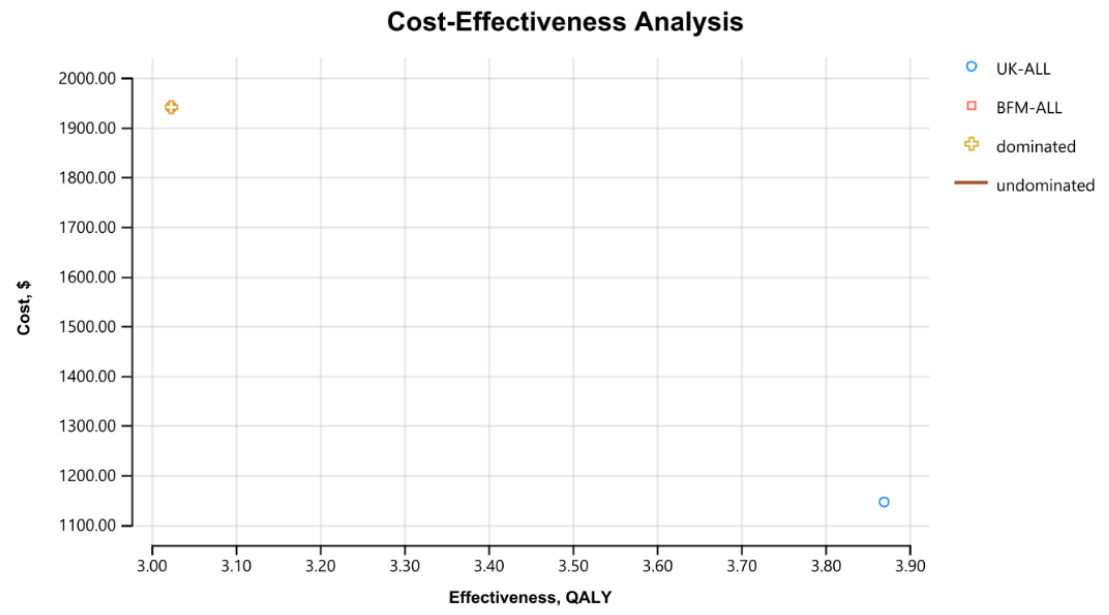

Figure 3 cost effectiveness analysis of UK-ALL and BFM-ALL

As shown in Figure 3, the UK-ALL is complete dominant and BFM-ALL is dominated.

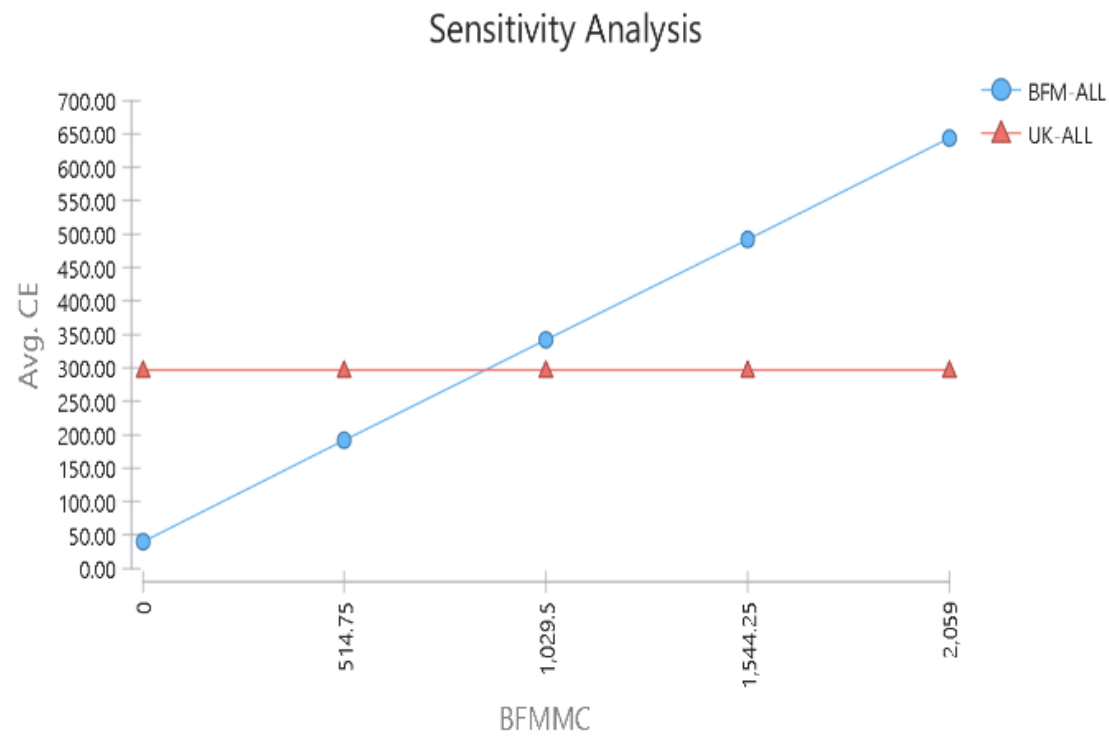




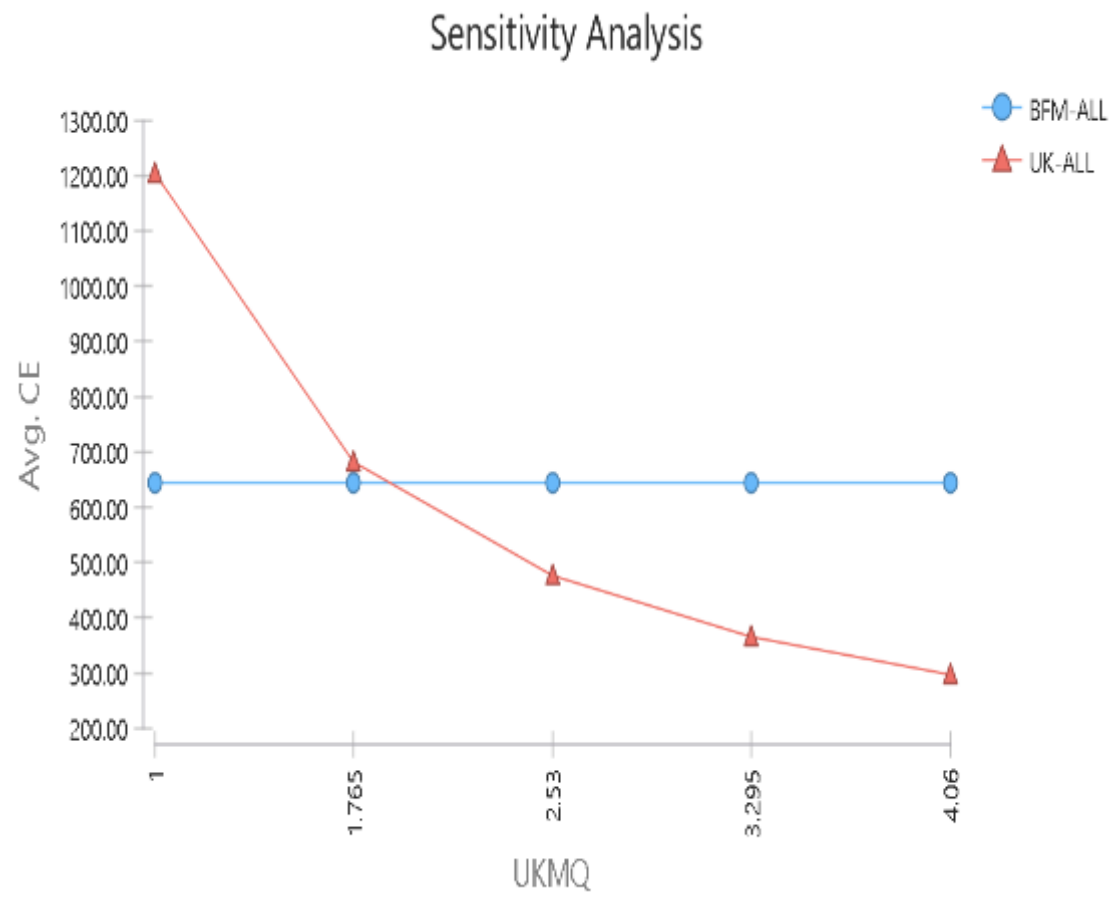

Figure 4 sensitivity analysis BFM maintenance cost and UK maintenance QALY

Based on high effects of two variable's BFM maintenance cost (BFMMC) and UK maintenance QALY (UKMQ)on the cost effectiveness ratio, sensitivity analysis was done, so Figure 4 shows up to twice the variables must be changed to change the results.

The calculations indicate that the rate of complete recovery after 5 years from start of treatment in UK-ALL is greater than BFM-ALL. So, the BFM-ALL protocols have a higher overall cost and a lower impact than UK-ALL, in other words, the UK-ALL is dominant and BFM-ALL is dominated. The sensitivity analysis shows that the results can be sensitive in the absence of a 50\% QALY BFM protocol and don't show any sensitivity in less than this value.

\section{DISCUSSION}

This study is the first study available to comprehensive examine and compare the cost and consequences in term of quality of life and years of life among children with all UK-ALL BFM-ALL, therefore the final analysis showed that achievement of 5 years treatment in UK-ALL is greater than BFM-ALL[13]. Given their somewhat equally similar outcomes, they have a great influence on the dominance of this method. Various studies have made acute childhood lymphoblastic leukemia (ALL) one of the most treatable human cancers $[14,15]$ and this study approves this results. But in this regard Rae who examined the utility cost of all children with two BFM and DFCL protocols in Canada and several European countries in terms of quality of life and calculating QALY for 5 years period showed that the average direct cost of treatment for patients the BFM method is $\$ 88480$ also in the BFM protocols the QALY score of 5 years the average QALY between 0.7 to 0.8 was obtained $[15,16]$. The QALY of this study is similar to other protocols but its varied in costs relatively [17, 18]. Similar studies among other worlds countries showed that in European countries and USA the cost is much higher than the cost of similar protocols in other Asian and American countries [19], but based on the demographic characteristics in table 4-3 the average age of the children is about 9 years and the incidence and prevalence of the disease in hoys is always more than of girls which is similar in other studies[10]. the average length of hospitalization is an important factor influencing 
the cost of treatment as this study indicates a significant difference in the cost difference between the two protocols. The results of this study confirm that, as a general principle, the cost of treatment in developing countries differs from developed countries[4, 10, 15]. Maybe the most important reason refers the health economic conditions of these countries. In this study, the costs of hospital services include catering, food and cleaning are the least significant factors affecting total costs. In general researches performed in similar studies among other countries showed that in developed countries, the costs are much higher than the cost of similar protocols in other developing countries. But the remarkable point is the dramatic treatment of this disease which has changed from an incurable disease to a treatable disease in most protocols $[8,11,15$, 17, 20, 21]. Therefore, the debatable issue is choosing the best option among the existing protocols with the best cost-effectiveness, so economic evaluation methods[17], especially the decision tree, can help to choose best option for treatment.

\section{Conclusions}

Because the BFM-ALL protocol has a higher overall cost and a lower impact than UK-ALL therefore the UK-ALL is dominant and BFM-ALL is a dominated. considering significant different between cost of these two protocols and even assuming that their consequences are constant and insignificant, we could say, the UK-ALL is the better protocol to use but ultimate decision made by final decision maker and doctors who make a specific decision based on contingency conditions. so, policymakers and oncologist should advocate the economic evaluation methods in the treatment of patients to save resources beside treating patients like UK-ALL.

\section{Limitations of the study}

The most important issue in economic evaluation studies is adjusting of money with consideration of time and high inflation rate in developing countries.

\section{CONFLICT OF INTEREST}

The authors declare that there is no conflict of Interest

\section{ACKNOWLEDGMENTS}

The authors would like to acknowledge consultants working in the Hepatitis Research Center in Lorestan university of medical sciences .

\section{References}

1. Chen, S.-L., et al., Toward the Cure of Acute Lymphoblastic Leukemia in Children in China. JCO Global Oncology, 2021. 7 : p. 1176-1186.

2. Hayati, H., et al., Cost-Analysis of Treatment of Childhood Acute Lymphoblastic Leukemia Based on UKALL Protocol. Iranian Journal of Pediatrics, 2018. 28 (6): p. e7985.

3. Conter, V., et al., Long-term results of the Italian Association of Pediatric Hematology and Oncology (AIEOP) Studies 82, 87, 88, 91 and 95 for childhood acute lymphoblastic leukemia. Leukemia, 2010. 24 (2): p. $255-264$.

4. Hayati, H., et al., Cost-Utility of protocols of BFM-ALL and UK-ALL for treatment of children with acute lymphoblastic leukemia in Iran. Iranian journal of public health, 2018. 47 (3): p. 407.

5. Schrappe, M., et al., Long-term results of four consecutive trials in childhood ALL performed by the ALL-BFM study group from 1981 to 1995. Leukemia, 2000. 14 (12): p. 2205-2222.

6. Duncan, K. and E. Sinanovic, A cost comparison analysis of paediatric intermediate care in a tertiary hospital and an intermediate care facility in Cape Town, South Africa. Plos one, 2019.14 (4): p. e0214492.

7. Doshmangir, L., et al., Incidence of Catastrophic Health Expenditure and Its Determinants in Cancer Patients: A Systematic Review and Meta-analysis. Applied Health Economics and Health Policy, 2021.19 
(6): p. 839-855.

8. James, S.L., et al., Global, regional, and national incidence, prevalence, and years lived with disability for 354 diseases and injuries for 195 countries and territories, 1990-2017: a systematic analysis for the Global Burden of Disease Study 2017. The Lancet, 2018.392 (10159): p. 1789-1858.

9. Myles, A.J., et al., An introduction to decision tree modeling. Journal of Chemometrics: A Journal of the Chemometrics Society, 2004. 18 (6): p. 275-285.

10. Fung, A., et al., Cost and cost-effectiveness of childhood cancer treatment in low-income and middleincome countries: a systematic review. BMJ global health, 2019. 4 (5): p. e001825.

11. Liu, A.P.-Y., et al., Treatment barriers and clinical outcome of children with medulloblastoma in China: a report from the Chinese Children's Cancer Group (CCCG). Neuro-oncology advances, 2021.3 (1): p. vdab134.

12. Yang, W., et al., Pulse therapy with vincristine and dexamethasone for childhood acute lymphoblastic leukaemia (CCCG-ALL-2015): an open-label, multicentre, randomised, phase 3, non-inferiority trial. The Lancet Oncology, 2021. 22 (9): p. 1322-1332.

13. Bartram, J., P. Veys, and A. Vora, Improvements in outcome of childhood acute lymphoblastic leukaemia (ALL) in the UK-a success story of modern medicine through successive UKALL trials and international collaboration. British Journal of Haematology, 2020. 191 (4): p. 562-567.

14. Pui, C.-H., et al., Global efforts toward the cure of childhood acute lymphoblastic leukaemia. The Lancet Child \& Adolescent Health, 2018. 2 (6): p. 440-454.

15. Rae, C., et al., Economic evaluation of treatment for acute lymphoblastic leukaemia in childhood. European journal of cancer care, 2014. 23 (6): p. 779-785.

16. Fardell, J.E., et al., Health-related quality of life of children on treatment for acute lymphoblastic leukemia: a systematic review. Pediatric Blood \& Cancer, 2017. 64 (9): p. e26489.

17. McGrady, M.E., et al., Cost-effectiveness analysis of an adherence-promotion intervention for children with leukemia: a Markov model-based simulation. Journal of pediatric psychology, 2018.43 (7): p. 758-768.

18. Maser, B., et al., Levofloxacin prophylaxis in hospitalized children with leukemia: A cost-utility analysis. Pediatric Blood \& Cancer, 2020. 67 (10): p. e28643.

19. Sruamsiri, R., et al., What are the volume and budget needs to provide chemotherapy to all children with acute lymphoblastic leukaemia in Thailand? Development and application of an estimation tool. BMJ open, 2020. 10 (10): p. e041901.

20. Gupta, S., et al., Health care utilisation and costs associated with different treatment protocols for newly diagnosed childhood acute lymphoblastic leukaemia: A population-based study in Ontario, Canada. European Journal of Cancer, 2021. 151 : p. 126-135.

21. Möricke, A., et al., Long-term results of five consecutive trials in childhood acute lymphoblastic leukemia performed by the ALL-BFM study group from 1981 to 2000. Leukemia, 2010. 24 (2): p. 265-284. 